\title{
A REMARK ON RANDOM PERTURBATIONS OF THE NONLINEAR PENDULUM
}

\author{
By MARK FreidLIN ${ }^{1}$ AND MATTHIAS WebER \\ University of Maryland and Technische Universität Dresden
}

We describe the long-time behavior of the nonlinear pendulum perturbed by a small noise. To derive this asymptotics, one has to consider diffusion processes on the graph corresponding to the Hamiltonian of the pendulum.

1. Introduction. Statement of the problem. We considered in [6] random perturbations of an oscillator with one degree of freedom,

$$
\ddot{\tilde{X}}_{t}^{\varepsilon}+f\left(\tilde{X}_{t}^{\varepsilon}\right)=\sqrt{\varepsilon} \dot{W}_{t}, \quad \tilde{X}_{0}^{\varepsilon}=x, \quad \dot{\tilde{X}}_{0}^{\varepsilon}=y,
$$

where $W_{t}$ is the Wiener process in $R^{1}$. It was shown that after a proper time rescaling, the slow component of the process converges to a diffusion process on the graph corresponding to the Hamiltonian of system (1.1). Formulated in more detail, if $\Gamma$ is the graph homeomorphic to the set of connected components of the level sets of the Hamiltonian,

$$
H(x, y)=\frac{y^{2}}{2}+F(x), \quad F^{\prime}(x)=f(x),
$$

provided with the natural topology, and $Y: R^{2} \rightarrow \Gamma$ is the mapping such that $Y(x, y),(x, y) \in R^{2}$, is the point of $\Gamma$ corresponding to the connected component of the level set containing the trajectory of the nonperturbed system starting at $(x, y)$, then $Y\left(\tilde{X}_{t / \varepsilon}^{\varepsilon}, \dot{\tilde{X}}_{t / \varepsilon}^{\varepsilon}\right)$ converges weakly in the space of continuous functions on $[0, T]$, for any $0<T<\infty$, to a diffusion process $Z_{t}$ on $\Gamma$ as $\varepsilon \downarrow 0$. We calculated the characteristics of $Z_{t}$, which are the differential operators defining the behavior of $Z_{t}$ inside the edges and gluing conditions at the vertices. This result allows calculating the main terms as $\varepsilon \downarrow 0$ of many interesting characteristics of the perturbed system. Locally, in the neighborhood of the noncritical values of the Hamiltonian, the limiting behavior of the slow component can be, actually, derived from Khas'minskii's results [8].

The results in [6] were obtained under the assumption that $f(x), x \in R^{1}$, is a smooth generic function: if $f\left(x_{0}\right)=0$ then $f^{\prime}\left(x_{0}\right) \neq 0$, and if $f\left(x_{0}\right)=$ $f\left(x_{1}\right)=0$ then $F\left(x_{0}\right) \neq F\left(x_{1}\right)$, and $\lim _{|x| \rightarrow \infty} F(x)=\infty$. But the most popular equation of such kind, the equation of a pendulum, has the form

$$
\ddot{X}_{t}+\sin X_{t}=0,
$$

Received November 1997; revised December 1998.

${ }^{1}$ Supported by NSF Grant DMS-95-04177.

AMS 1991 subject classifications. 60H10, 34C29, 35B20.

Key words and phrases. Averaging principle, random perturbations, nonlinear pendulum. 
and it does not satisfy the assumptions. In this paper we study perturbations of (1.2). We also consider a more general class of perturbations which arise in applications.

Namely, we study the process

$$
\ddot{\tilde{X}}_{t}^{\varepsilon}+\sin \tilde{X}_{t}^{\varepsilon}=\varepsilon b\left(\tilde{X}_{t}^{\varepsilon}, \dot{\tilde{X}}_{t}^{\varepsilon}\right)+\sqrt{\varepsilon} \dot{W}_{t},
$$

where $b(x, y)$ is $2 \pi$-periodic in the variable $x$. There are at least two problems where the addition of the term $\varepsilon b(x, y)$ is of interest. One example is a model of phase synchronization arising in radiophysics. In this problem $b(x, y)=$ $-\kappa y+\gamma$, and the term $\varepsilon \kappa$ is interpreted as the "initial detuning" of the system. It was noted in [1] that the slow component in this problem should be considered on the graph, although the limiting process was not described mathematically. We will do this here. The results allow calculating a number of interesting characteristics of the system.

A second example concerns the optimal stabilization: after the time rescaling $\tilde{X}_{t / \varepsilon}^{\varepsilon}=X_{t}^{\varepsilon}$, one can rewrite the perturbed equation as the system

$$
\begin{aligned}
& \dot{X}_{t}^{\varepsilon}=\frac{1}{\varepsilon} Y_{t}^{\varepsilon}, \\
& \dot{Y}_{t}^{\varepsilon}=-\frac{1}{\varepsilon} \sin X_{t}^{\varepsilon}+\dot{W}_{t}+b\left(X_{t}^{\varepsilon}, Y_{t}^{\varepsilon}\right) .
\end{aligned}
$$

Let $G$ be a domain in $R^{2}$ such that the physical system, described by (1.4), is "alive" until the time when $\left(X_{t}^{\varepsilon}, Y_{t}^{\varepsilon}\right)$ leaves $G$. This means that the system dies at the moment $\tau^{\varepsilon, b}=\inf \left\{t:\left(X_{t}^{\varepsilon}, Y_{t}^{\varepsilon}\right) \notin G\right\}$. The case of a domain $G$ bounded by trajectories of the nonperturbed system is of interest. Suppose we want to choose the control $b(x, y)$ from a certain set $\Pi$ such that $E_{x, \dot{x}} \tau^{\varepsilon, b}$ is as large as possible. Of course, if the set $\Pi$ is not very bad, and $b^{*}$ denotes the optimal control, one can write a Bellman equation for $V(x, y)=E_{x, y} \tau^{\varepsilon, b^{*}}$. However, it will be a degenerate nonlinear equation depending on a small parameter and even to find the numerical solution of such an equation is difficult. The results of this paper allow calculating the main term of $u^{\varepsilon}(x, y)=E_{x, y} \tau^{\varepsilon, b}$ as $\varepsilon \downarrow 0$ in a rather explicit form. Then one can choose $b^{* *}(x, y) \in \Pi$ which maximizes the main term. Such a control, although it is not optimal for fixed $\varepsilon$, is not worse then any other control if $\varepsilon$ is small enough. An interesting feature of this asymptotically optimal control is that it is a bang-bang type control with several switching points; compare with [2].

And, finally, one more remark: we consider here white noise type perturbations. Suppose the process in the right side of (1.1) is $\sqrt{\varepsilon} \zeta_{t}$ where $\zeta_{t}$ is a mean zero stationary process with good enough mixing properties. After the change of time, the system for the perturbed process has the form

$$
\begin{aligned}
& \dot{X}_{t}^{\varepsilon}=\frac{1}{\varepsilon} Y_{t}^{\varepsilon}, \\
& \dot{Y}_{t}^{\varepsilon}=-\frac{1}{\varepsilon} f\left(X_{t}^{\varepsilon}\right)+\frac{1}{\sqrt{\varepsilon}} \zeta_{t / \varepsilon} .
\end{aligned}
$$


Under the assumptions on $\zeta_{t}$ the process

$$
\frac{1}{\sqrt{\varepsilon}} \int_{0}^{t} \zeta_{s / \varepsilon} d s=\sqrt{\varepsilon} \int_{0}^{t / \varepsilon} \zeta_{s} d s
$$

converges to $\mathrm{K} \tilde{W}_{t}$, where $\tilde{W}_{t}$ is a Wiener process and $\mathrm{K}$ is a constant which can be expressed through the correlation function of $\zeta_{t}$. We can assume without loss of generality that $\mathrm{K}=1$.

These arguments allow the expectation that the solution of (1.5), for $0<\varepsilon \ll 1$, behaves like the solution of (1.4) with an appropriate drift term.

2. Main result. Consider the equation of a randomly perturbed pendulum,

$$
\ddot{\tilde{X}}_{t}^{\varepsilon}+\sin \tilde{X}_{t}^{\varepsilon}=\varepsilon b\left(\tilde{X}_{t}^{\varepsilon}, \dot{\tilde{X}}_{t}^{\varepsilon}\right)+\sqrt{\varepsilon} \dot{W}_{t}
$$

$\left(\tilde{X}_{0}^{\varepsilon}, \dot{X}_{0}^{\varepsilon}\right)=(x, y), x \in[0,2 \pi]$, where $W_{t}$ is the Wiener process in $R^{1}, \varepsilon$ is a small positive parameter, $b$ is periodic and uniformly Lipschitz,

$$
\begin{aligned}
b(x, y) & =b(x+2 \pi, y),(x, y) \in R^{2} \\
\left|b\left(x_{1}, y_{1}\right)-b\left(x_{2}, y_{2}\right)\right| & \leq L_{b}\left(\left(x_{1}-x_{2}\right)^{2}+\left(y_{1}-y_{2}\right)^{2}\right)^{1 / 2},
\end{aligned}
$$

$\left(x_{i}, y_{i}\right) \in R^{2}, i=1,2, L_{b}>0$. The process $\tilde{X}_{t}^{\varepsilon}$ should be considered modulo $2 \pi$.

It is convenient to draw the phase picture of the pendulum [see Figure 1(c)] on the cylinder [Figure 1(a)].

The trajectories between the curves crossing at $\mathrm{O}_{2}$ correspond to the oscillations. The trajectories below and above this domain correspond to rotations in one and in the other direction.

In [1] this model was considered for $b(x, y)=-\kappa y+\gamma$ with real parameters $\kappa$ and $\gamma$.

Introducing the Hamilton function,

$$
H(x, y)=-\cos x+\frac{1}{2} y^{2},
$$

(2.1) can be written as the system

$$
\begin{aligned}
& \dot{\tilde{X}}_{t}^{\varepsilon}=H_{y}\left(\tilde{X}_{t}^{\varepsilon}, \tilde{Y}_{t}^{\varepsilon}\right)=\tilde{Y}_{t}^{\varepsilon}, \\
& \dot{\tilde{Y}}_{t}^{\varepsilon}=-H_{x}\left(\tilde{X}_{t}^{\varepsilon}, \tilde{Y}_{t}^{\varepsilon}\right)+\varepsilon b\left(\tilde{X}_{t}^{\varepsilon}, \tilde{Y}_{t}^{\varepsilon}\right)+\sqrt{\varepsilon} \dot{W}_{t},
\end{aligned}
$$

$\tilde{X}_{0}^{\varepsilon}=x, \tilde{Y}_{0}^{\varepsilon}=y$.

After the time rescaling $X_{t}^{\varepsilon}=\tilde{X}_{t / \varepsilon}^{\varepsilon}, Y_{t}^{\varepsilon}=\tilde{Y}_{t / \varepsilon}^{\varepsilon}$, that system becomes

$$
\begin{aligned}
& \dot{X}_{t}^{\varepsilon}=\frac{1}{\varepsilon} H_{y}\left(X_{t}^{\varepsilon}, Y_{t}^{\varepsilon}\right)=\frac{1}{\varepsilon} Y_{t}^{\varepsilon}, \\
& \dot{Y}_{t}^{\varepsilon}=-\frac{1}{\varepsilon} H_{x}\left(X_{t}^{\varepsilon}, Y_{t}^{\varepsilon}\right)+b\left(X_{t}^{\varepsilon}, Y_{t}^{\varepsilon}\right)+\dot{W}_{t},
\end{aligned}
$$



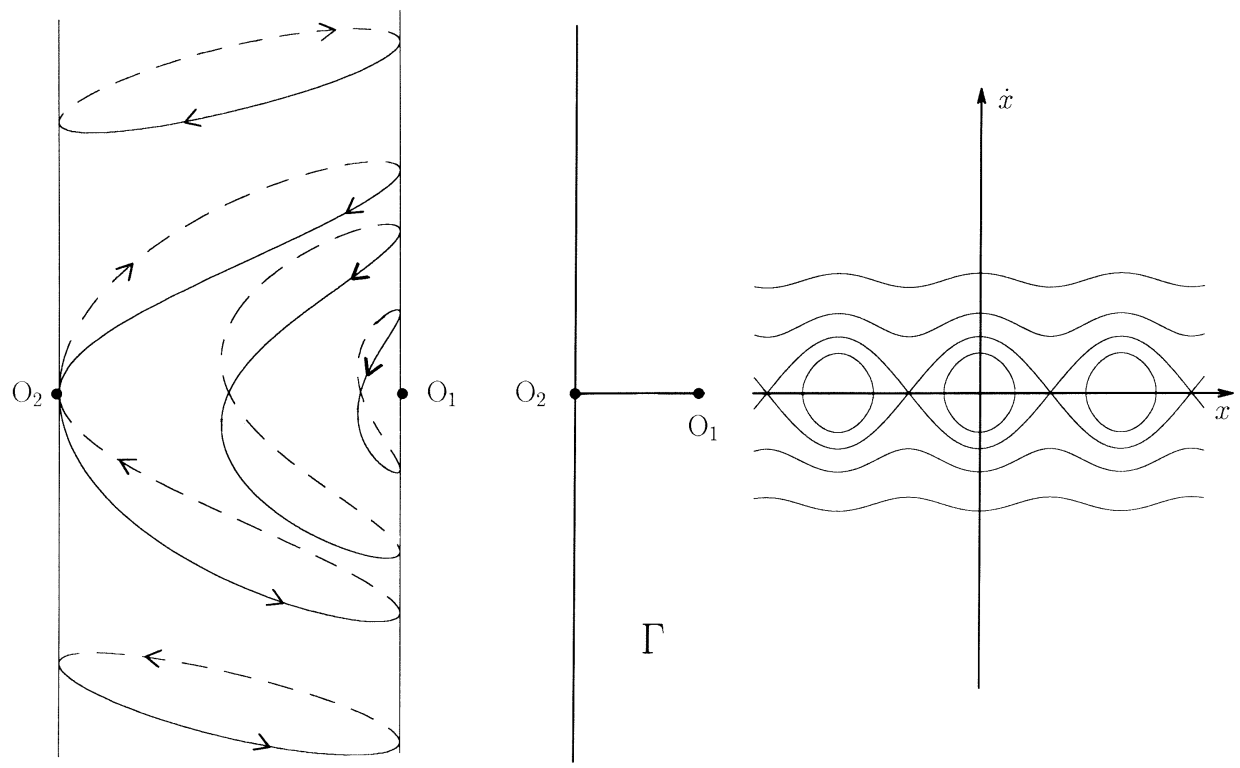

FIG. 1.

$X_{0}^{\varepsilon}=x, Y_{0}^{\varepsilon}=y$. This process is governed by the operator

$$
L^{\varepsilon} f(x, y)=\frac{1}{2} f_{y y}(x, y)+\frac{1}{\varepsilon}\left(y f_{x}(x, y)-\sin x f_{y}(x, y)\right)+b(x, y) f_{y}(x, y) .
$$

For $b=0$, the system (2.2), (2.3) can be regarded as a degenerate perturbation by white noise of the system

$$
\begin{aligned}
& X_{t}(x, y)=H_{y}\left(X_{t}(x, y), Y_{t}(x, y)\right) \\
& Y_{t}(x, y)=-H_{x}\left(X_{t}(x, y), Y_{t}(x, y)\right),\left(X_{0}(x, y), Y_{0}(x, y)\right)=(x, y) .
\end{aligned}
$$

Such perturbations are considered in [6]. Although the Hamiltonian function $H$ is not generic here, all considerations of [6] hold also here for $b \equiv 0$. This is easy to check. If we consider the Hamiltonian of the pendulum on a cylinder [see Figure 1(a)] we can use the same arguments as in [6] (see also [7]).

We introduce the graph $\Gamma$ [see Figure 1(b)] which is homeomorphic to the set of connected components of the level sets of the Hamiltonian $H(x, y)$ [Figure 1(c)]. This graph consists of three edges $I_{1}, I_{2}, I_{3}$, one interior vertex $O_{2}$ which connects these three edges and an exterior vertex $O_{1}$ at the opposite end of $I_{1}$. We introduce the mapping,

$$
Y: R^{2} \rightarrow \Gamma
$$

such that:

1. For any point $(x, y)$ with $H(x, y)<1$, we have $Y(x, y) \in I_{1}$ and $\operatorname{dist}\left(O_{2}, Y(x, y)\right)=1-H(x, y)$, and 
2. For any point $(x, y)$ with $H(x, y)>1$ and $y>0(y<0)$, we have $Y(x, y) \in$ $I_{2}\left(Y(x, y) \in I_{3}\right), \operatorname{dist}\left(O_{2}, Y(x, y)\right)=H(x, y)-1$.

Here $\operatorname{dist}\left(z_{1}, z_{2}\right)$ is the Euclidean distance of two points $z_{1}, z_{2}$ on an edge of $\Gamma$. If $H(x, y)=1$ then $Y(x, y)=O_{2}$; if $H(x, y)=-1$ then $Y(x, y)=O_{1}$. The edge $I_{1}$ is of length 2 as $\operatorname{dist}\left(O_{1}, O_{2}\right)=2$. The other two edges are of infinite length. A coordinate system on $\Gamma$ is given by $(H(x, y), i)$ if $Y(x, y) \in I_{i}$.

As in [6] and [7], it turns out that the processes $Y\left(X_{t}^{\varepsilon}, Y_{t}^{\varepsilon}\right)$ for $\varepsilon \downarrow 0$ tend to a diffusion process on $\Gamma$ given by a differential operator on each edge of $\Gamma$ and a gluing condition at the vertex $\mathrm{O}_{2}$ describing the domain of the generator. Let

$$
T(z)=\int_{-x_{0}}^{x_{0}} \frac{d x}{\sqrt{2 z+2 \cos x}}, \quad z \neq 1,
$$

$x_{0}=\arccos (-z)$ if $z<1$, and $x_{0}=\pi$ if $z>1$. This is the period of the deterministic trajectories corresponding to the value $z$ of the Hamiltonian if $z>1$, and it is one half of that period if $z<1$. Let

$$
S(z)=\int_{-x_{0}}^{x_{0}} \sqrt{2 z+2 \cos x} d x .
$$

Thus, $S(z)$ is one half of the area on the cylinder encircled by $C(z)$, if $z<1$, and it is one half of the area on the cylinder bounded by the two components of $C(z)$, if $z>1$. We now state the result.

THEOREM 1. Let $\left(X_{t}^{\varepsilon}, Y_{t}^{\varepsilon} ; P_{x, y}^{\varepsilon}\right)$ be the diffusion process on $R^{2}$ corresponding to the differential operator,

$$
\begin{aligned}
L^{\varepsilon} f(x, y)= & \frac{1}{2} f_{y y}(x, y)+\frac{1}{\varepsilon}\left(y f_{x}(x, y)-\sin x f_{y}(x, y)\right) \\
& +b(x, y) f_{y}(x, y) .
\end{aligned}
$$

Then the distributions of the processes $Y\left(X_{t}^{\varepsilon}, Y_{t}^{\varepsilon}\right)$ in the space of continuous functions $\varphi:[0, T] \rightarrow \Gamma$ for any $T>0$ with respect to $P_{x, y}^{\varepsilon}$ converge weakly as $\varepsilon \downarrow 0$ to the probability measure $P_{Y(x, y)}$, where $\left(y(t), P_{y}\right)$ is the process on the graph $\Gamma$ defined by operators $L_{i}$,

$$
\begin{aligned}
L_{i} v_{i}(z)= & \frac{1}{2 T(z)}\left(\frac{d}{d z} S(z) v_{i}^{\prime}(z)\right) \\
& \pm \frac{\int_{-x_{0}}^{x_{0}} b(x, \pm \sqrt{2 z+2 \cos x}) d x}{\int_{-x_{0}}^{x_{0}}(d x / \sqrt{2 z+2 \cos x})} v_{i}^{\prime}(z), \quad i=1,2,3,
\end{aligned}
$$

on each edge $I_{i}$. Here "+" is taken if $i=2$, and "-" is taken if $i=3$; the mean value of the "+" and the "-" expression should be taken if $i=1$. The parameter $x_{0}$ is equal to $\pi$ if $i=2,3$, and it is equal to $\arccos (-z)$ if $i=1$. The gluing condition is

$$
2 v_{1}^{\prime}(1)=v_{2}^{\prime}(1)+v_{3}^{\prime}(1)
$$


The functions $v_{i}$ on $\Gamma$ should be continuous as well as the functions $L_{i} v_{i}$, $i=1,2,3$.

As discussed above, we can get the result for $b \equiv 0$ from [6].

LEMMA 2.1. Let $\left(X_{t}^{0, \varepsilon}, Y_{t}^{0, \varepsilon} ; P_{x, y}^{0, \varepsilon}\right)$ be the diffusion process on $R^{2}$ corresponding to the differential operator

$$
L^{0, \varepsilon} f(x, y)=\frac{1}{2} f_{y y}(x, y)+\frac{1}{\varepsilon}\left(y f_{x}(x, y)-\sin x f_{y}(x, y)\right) .
$$

Then the statement of Theorem 1 holds with $b \equiv 0$.

Now we consider the case $b \not \equiv 0$. First we show that the shape of the operators governing the limiting diffusions inside the edges of the graph can be obtained using the standard averaging procedure. We follow the lines of [7] and [6]. Denote $x=(x, y), \underline{X}_{t}^{\varepsilon}=\left(X_{t}^{\varepsilon}, Y_{t}^{\varepsilon}\right), \tilde{X}_{t}^{\varepsilon}=\left(\tilde{X}_{t}^{\varepsilon}, \tilde{Y}_{t}^{\varepsilon}\right)$ and $X_{t}(x)=$ $\left(X_{t}(\underline{x}), Y_{t}(\underline{x})\right)$. In the following we denote by $A_{i}, i=1,2, \ldots$, positive constants.

LEMMA 2.2. Let $K$ be a bounded domain in $R^{2}$. Then for small $\varepsilon$,

$$
P_{\underline{x}}^{\varepsilon}\left\{\sup _{0<t<T}\left|\tilde{X}_{t}^{\varepsilon}-\underline{X}_{t}(\underline{x})\right| \geq \eta\right\} \leq A(K, k)\left(\exp \left(A_{K} T\right)-1\right)^{k} \frac{\varepsilon^{k}}{\eta^{2 k}},
$$

$k=1,2, \ldots$, where $A_{K}$ and $A(K, \cdot)$ depend on the Lipschitz constants of the functions $\nabla H$ and $b$ and on the bound of $|b|$ on $K$, and $\eta$ is an arbitrary positive number.

PROOF. We only sketch the proof because most of it is standard (cf. Lemma 4.2 of [7]). Itô's formula gives for $\left|\underline{\tilde{X}}_{T}^{\varepsilon}-\underline{X}_{T}(\underline{x})\right|^{2}$,

$$
\begin{aligned}
\left|\underline{X}_{T}^{\varepsilon}-\underline{X}_{T}(\underline{x})\right|^{2}= & 2 \int_{0}^{T}\left(\underline{X}_{t}^{\varepsilon}-\underline{X}_{t}(\underline{x})\right)\left(\bar{\nabla} H\left(\underline{X}_{t}^{\varepsilon}\right)-\bar{\nabla} H\left(\underline{X}_{t}(\underline{x})\right)\right) d t \\
& +2 \varepsilon \int_{0}^{T}\left(\tilde{Y}_{t}^{\varepsilon}-Y_{t}(\underline{x})\right) b\left(\underline{X}_{t}^{\varepsilon}\right) d t+\varepsilon T \\
& +2 \sqrt{\varepsilon} \int_{0}^{T}\left(\tilde{Y}_{t}^{\varepsilon}-Y_{t}(\underline{x})\right) d W_{t} \\
\leq & 2 L_{H} \int_{0}^{T}\left|\underline{X}_{t}^{\varepsilon}-\underline{X}_{t}(\underline{x})\right|^{2} d t+\varepsilon T \\
& +2 \varepsilon \int_{0}^{T}\left|\tilde{Y}_{t}-Y_{t}(\underline{x})\right|\left|b\left(\underline{X}_{t}^{\varepsilon}\right)-b\left(\underline{X}_{t}(\underline{x})\right)\right| d t \\
& +2 \varepsilon \int_{0}^{T}\left|\tilde{Y}_{t}^{\varepsilon}-Y_{t}(\underline{x})\right|\left|b\left(\underline{X}_{t}(\underline{x})\right)\right| d t
\end{aligned}
$$




$$
\begin{aligned}
& +2 \sqrt{\varepsilon} \int_{0}^{T}\left(\tilde{Y}_{t}^{\varepsilon}-Y_{t}(\underline{x})\right) d W_{t} \\
\leq & 2\left(L_{H}+\varepsilon L_{b}+\varepsilon B_{K}\right) \int_{0}^{T}\left|\underline{X}_{t}^{\varepsilon}-\underline{X}_{t}(\underline{x})\right|^{2} d t \\
& +\varepsilon T\left(1+2 B_{K}\right)+2 \sqrt{\varepsilon} \int_{0}^{T}\left(\tilde{Y}_{t}^{\varepsilon}-Y_{t}(\underline{x})\right) d W_{t} \\
\leq & A_{1} \int_{0}^{T}\left|\underline{X}_{t}^{\varepsilon}-\underline{X}_{t}(\underline{x})\right|^{2} d t+A_{2} \varepsilon T \\
& +\sqrt{\varepsilon} \int_{0}^{T}\left(\tilde{Y}_{t}^{\varepsilon}-Y_{t}(\underline{x})\right) d W_{t},
\end{aligned}
$$

if $\varepsilon$ is small enough. Here $L_{H}$ denotes the Lipschitz constant of $\bar{\nabla} H$, and $B_{K}$ is a bound for $|b|$ on $K$. The constants $A_{1}, A_{2}$ depend on $K$. Let $Z_{T}^{\varepsilon}$ denote the process defined by the right-hand side of (2.10). Then $Z_{T}^{\varepsilon}$ is a positive submartingale, and we get with the Gronwall-Bellman inequality,

$$
\begin{aligned}
Z_{T}^{\varepsilon} & \leq A_{1} \int_{0}^{T} Z_{t}^{\varepsilon} d t+A_{2} \varepsilon T+\sqrt{\varepsilon} \int_{0}^{T}\left(\tilde{Y}_{t}^{\varepsilon}-Y_{t}(\underline{x})\right) d W_{t} \\
E_{\underline{x}} Z_{T}^{\varepsilon} & \leq A_{1} \int_{0}^{T} E_{\underline{x}} Z_{t}^{\varepsilon} d t+A_{2} \varepsilon T \\
& \leq A_{2} \varepsilon \int_{0}^{T} \exp \left\{A_{1}(T-t)\right\} d t \leq A_{3} \varepsilon\left(\exp \left(A_{1} T\right)-1\right) .
\end{aligned}
$$

Kolmogorov's inequality implies the statement of the lemma for $k=1$. The proof is similar for $k>1$.

REMARK. To calculate the shape of the operators inside the edges, only estimates inside bounded (in $y$-coordinate) domains are needed. We can therefore change the function $b$ outside such domains to get the estimate of Lemma 2.2 uniformly for all $x \in R^{2}$. But, if $|b|$ is bounded, one can get the estimate of Lemma 2.2 independent of a Lipschitz constant of $b$. Thus, one can also consider bounded drift functions $b$ with a finite number of discontinuities.

Using that lemma it is easy to check that Lemmas 4.3 and 4.4 of [7], as well as Lemmas 2.2.3 and 2.2.4 of [6], can be proved analogously as in the cited papers, although some of the constants in these proofs need to be larger due to the additional drift. Then the results corresponding to Lemmas 4.8 and 4.9 of [7] can be shown. In the proof of the result corresponding to Lemma 4.9 of [7], the application of Itô's formula gives a different result in the situation here: for the Markov time $\tau=\tau_{i}^{\varepsilon}\left(H_{1}, H_{2}\right)$ of first exit of the process $\underline{X}_{t}^{\varepsilon}$ from a region $D_{i}\left(H_{1}, H_{2}\right)$,

$$
D_{i}\left(H_{1}, H_{2}\right)=\left\{\underline{x} \in[-\pi, \pi] \times R^{1}, H_{1}<H(\underline{x})<H_{2}, Y(\underline{x}) \in I_{i}\right\},
$$


we get for $\underline{x} \in D_{i}\left(H_{1}, H_{2}\right), \lambda>0$, and smooth functions $f$, by Itô's formula

$$
\begin{aligned}
E_{\underline{x}} \exp (-\lambda \tau) f\left(H\left(\underline{X}_{\tau}^{\varepsilon}\right)\right)-f(H(x)) & \\
=E_{\underline{x}} \int_{0}^{\tau} \exp (-\lambda t) & {\left[-\lambda f\left(H\left(\underline{X}_{t}^{\varepsilon}\right)\right)+\frac{1}{2} f^{\prime \prime}\left(H\left(\underline{X}_{t}^{\varepsilon}\right)\right) H_{y}^{2}\left(\underline{X}_{t}^{\varepsilon}\right)\right.} \\
& \left.+\frac{1}{2} f^{\prime}\left(H\left(\underline{X}_{t}^{\varepsilon}\right)\right) H_{y y}\left(\underline{X}_{t}^{\varepsilon}\right)+f^{\prime}\left(H\left(\underline{X}_{\tau}^{\varepsilon}\right)\right) H_{y}\left(\underline{X}_{t}^{\varepsilon}\right) b\left(\underline{X}_{t}^{\varepsilon}\right)\right] d t .
\end{aligned}
$$

Following the arguments of the proof of Lemma 4.9 of [7], we get that the operator $L_{i}$, which governs the limiting diffusion inside the edge $I_{i}$ of $\Gamma$, now has the form

$$
\begin{aligned}
L_{i} v_{i}(z) & =L_{i}^{0} v_{i}(z)+B_{i}(z) v_{i}^{\prime}(z), \\
B_{i}(z) & =\frac{1}{\lambda(z)} \int_{C_{i}(z)} H_{y}(\underline{x}) b(\underline{x})|\nabla H(\underline{x})|^{-1} d l, \\
\lambda(z) & =\int_{C_{i}(z)}|\nabla H(\underline{x})|^{-1} d l,
\end{aligned}
$$

where the integration is taken over

$$
C_{i}(z)=\left\{\underline{x} \in[-\pi, \pi] \times R^{1}, H(\underline{x})=z, Y(\underline{x}) \in I_{i}\right\} .
$$

A simple calculation shows that the operators $L_{i}$ can be written as in (2.8).

The accessibility of the vertex $O_{2}$ and the inaccessibility of the vertex $O_{1}$ for that diffusion on $\Gamma$ follow from the corresponding properties of the diffusion with $b \equiv 0$, as shown in [6], and from the boundness of the additional drift for $b \not \equiv 0$ at these vertices [see (2.8)]. If $z$ tends to infinity, the operators $L_{i}$ behave like $A_{4} z v_{i}^{\prime \prime}+A_{5} z v_{i}^{\prime}, i=2,3$. It is easy to check that the point $z=\infty$ is a natural boundary for such diffusions (see [4]). Thus, no condition besides the boundness should be imposed on the functions $v_{i}, i=2,3$, for $z \rightarrow \infty$.

Now we show that the gluing conditions in the case $b \neq \equiv 0$ are the same as in the case $b \equiv 0$. This follows from the uniformly in $\varepsilon$ boundness of the Girsanov density and from the fact that the exit time from a narrow domain around the singular curve [with $H(\underline{x})=1$ ] is small for the limiting process.

Let $\underline{X}_{t}^{\varepsilon}$ be the process with $\operatorname{drift} b \not \equiv 0$. We denote by $\underline{X}_{t}^{0, \varepsilon}$ the corresponding process with $b \equiv 0$. Then, for any $T>0$, the measure $P_{\underline{x}}^{\varepsilon}$ of the process $\underline{X}_{t}^{\varepsilon}$ in the space of trajectories considered in $C(0, T)$ is absolutely continuous with respect to the measure $P_{\underline{x}}^{0, \varepsilon}$ of the process $\underline{X}_{t}^{0, \varepsilon}$, and the density $I_{0, T}^{\varepsilon}$ can be represented (see, e.g., [5]) as

$$
I_{0, T}^{\varepsilon}=\exp \left\{\int_{0}^{T} b\left(\underline{X}_{t}^{0, \varepsilon}\right) d W_{t}-\frac{1}{2} \int_{0}^{T} b^{2}\left(\underline{X}_{t}^{0, \varepsilon}\right) d t\right\} .
$$

To get the gluing conditions, we have to consider the process only in a bounded domain of $R^{2}$ containing the level set $C_{1}=\left\{\underline{x} \in[-\pi, \pi] \times R^{1}, H(\underline{x})=1\right\}$. We can therefore change the drift $b$ for large enough $|y|$ to get it bounded by a constant: $|b|<A_{6}$. In what follows, we will consider the process (denoted again 
by $\underline{X}_{t}^{\varepsilon}$ ) with that bounded drift. The gluing conditions will not be changed if we change $b$ in that way.

LEMMA 2.3. There exist constants $A_{7}>0, T_{0}>0$ such that for all $T<T_{0}$,

$$
E_{\underline{x}}\left(I_{0, T}^{\varepsilon}-1\right)^{2} \leq A_{7} T .
$$

Proof. Consider the process $Z_{t}^{\varepsilon}$,

$$
Z_{t}^{\varepsilon}=\int_{0}^{t} b\left(\underline{X}_{s}^{0, \varepsilon}\right) d W_{s}-\frac{1}{2} \int_{0}^{t} b^{2}\left(\underline{X}_{s}^{0, \varepsilon}\right) d s .
$$

By Itô's formula we get

$$
\begin{aligned}
d\left(I_{0, t}^{\varepsilon}-1\right)^{2}= & d\left(\exp \left\{Z_{t}\right\}-1\right)^{2} \\
= & 2\left(\exp \left\{2 Z_{t}\right\}-\exp \left\{Z_{t}\right\}\right) b\left(\underline{X}_{t}^{0, \varepsilon}\right) d W_{t} \\
& +\exp \left\{2 Z_{t}\right\} b^{2}\left(\underline{X}_{t}^{0, \varepsilon}\right) d t, \\
E_{\underline{x}}\left(I_{0, T}^{\varepsilon}-1\right)^{2}= & E_{\underline{x}} \int_{0}^{T} \exp \left\{2 Z_{t}\right\} b^{2}\left(\underline{X}_{t}^{0, \varepsilon}\right) d t \\
\leq & \left(A_{6}\right)^{2} E_{\underline{x}} \int_{0}^{T} \exp \left\{2 Z_{t}\right\} d t \\
= & A_{8} \int_{0}^{T} E_{\underline{x}} \exp \left\{\int_{0}^{t} 2 b\left(\underline{X}_{s}^{0, \varepsilon}\right) d W_{s}-\frac{1}{2} \int_{0}^{t}\left(2 b\left(\underline{X}_{s}^{0, \varepsilon}\right)\right)^{2} d s\right\} \\
& \times \exp \left\{\int_{0}^{t} b^{2}\left(\underline{X}_{s}^{0, \varepsilon}\right) d s\right\} d t \\
= & A_{8} \int_{0}^{T} E_{\underline{x}} \exp \left\{\int_{0}^{t} b^{2}\left(\underline{X}_{s}^{2 b, \varepsilon}\right) d s\right\} d t \\
\leq & A_{8} \int_{0}^{T} \exp \left\{\left(A_{6}\right)^{2} t\right\} d t \leq A_{9} T,
\end{aligned}
$$

for all $T$ from a bounded interval $\left[0, T_{0}\right]$. Here we used that the expression

$$
\exp \left\{\int_{0}^{t} 2 b\left(\underline{X}_{s}^{0, \varepsilon}\right) d W_{s}-\frac{1}{2} \int_{0}^{t}\left(2 b\left(\underline{X}_{s}^{0, \varepsilon}\right)\right)^{2} d s\right\}
$$

has the form of the Girsanov density if $b$ is replaced by $2 b$. Thus, $\underline{X}_{t}^{2 b, \varepsilon}$ denotes the respective process with drift $2 b$.

For small $\delta>0$ and for $i=1,2$, let

$$
\begin{aligned}
\tau_{i}^{\varepsilon}( \pm \delta) & =\inf \left\{t>0, \underline{X}_{t}^{\varepsilon} \notin D_{i}( \pm \delta)\right\}, \\
\tau_{i}^{0, \varepsilon}( \pm \delta) & =\inf \left\{t>0, \underline{X}_{t}^{0, \varepsilon} \notin D_{i}( \pm \delta)\right\}, \\
D_{i}( \pm \delta) & =\left\{\underline{x} \in[-\pi, \pi] \times R^{1},(-1)^{i}-\delta<H(\underline{x})<(-1)^{i}+\delta\right\} \\
C_{2 i}(\delta) & =\left\{\underline{x} \in \partial D_{2}( \pm \delta), Y(\underline{x}) \in I_{i}\right\} .
\end{aligned}
$$


LEMMA 2.4. For any positive $\lambda$ and $\kappa$ there exists $\delta_{0}>0$ such that for $0<\delta \leq \delta_{0}$ for sufficiently small $\varepsilon$ and for all $\underline{x} \in D_{2}( \pm \delta)$,

$$
E_{\underline{x}} \int_{0}^{\tau_{2}^{\varepsilon}( \pm \delta)} \exp (-\lambda t) d t<\kappa \delta
$$

and for all $\underline{x} \in D_{1}( \pm \delta)$,

$$
E_{\underline{x}} \int_{0}^{\tau_{1}^{\varepsilon}( \pm \delta)} \exp (-\lambda t) d t<\kappa
$$

Proof. We first mention that the statement is true for the process $\underline{X}^{0, \varepsilon}$ because the lemma corresponds to Lemma 3.4 of [7] which holds also in the degenerate case (see [6]). We can therefore find $\delta_{0}$ and $\varepsilon(\delta)$ such that

$$
E_{\underline{x}} \int_{0}^{\tau_{2}^{0, \varepsilon}( \pm \delta)} \exp (-\lambda t) d t<\kappa \delta / 2
$$

for all $\delta<\delta_{0}, \varepsilon<\varepsilon(\delta)$ and $\underline{x} \in D_{2}( \pm \delta)$.

Now let $\delta<\delta_{0}, \varepsilon<\varepsilon(\delta), \underline{x} \in D_{2}( \pm \delta)$ and $T<\min \left\{\kappa \delta / 4,\left(\kappa^{2} \delta^{2} \lambda^{2}\right) /\left(16 A_{7}\right)\right\}$. Using Lemma 2.3 and (2.14) we get

$$
\begin{aligned}
E_{\underline{x}} \int_{0}^{\tau_{2}^{\varepsilon}( \pm \delta)} \exp (-\lambda t) d t & \\
= & E_{\underline{x}}\left[\int_{0}^{\tau_{2}^{\varepsilon}( \pm \delta)} \exp (-\lambda t) d t ; \tau_{2}^{\varepsilon}( \pm \delta)<T\right] \\
& +E_{\underline{x}}\left[\int_{0}^{\tau_{2}^{\varepsilon}( \pm \delta)} \exp (-\lambda t) d t ; \tau_{2}^{\varepsilon}( \pm \delta) \geq T\right] \\
\leq & T+E_{\underline{x}} \int_{0}^{\tau_{2}^{0, \varepsilon}( \pm \delta)} \exp (-\lambda t) d t \\
& +E_{\underline{x}}\left[\left(I_{0, T}^{\varepsilon}-1\right) \int_{0}^{\tau_{2}^{0, \varepsilon}( \pm \delta)} \exp (-\lambda t) d t ; \tau_{2}^{0, \varepsilon}( \pm \delta) \geq T\right] \\
\leq & \frac{3}{4} \kappa \delta+\frac{1}{\lambda}\left[E_{\underline{x}}\left(I_{0, T}^{\varepsilon}-1\right)^{2}\right]^{1 / 2} \leq \kappa \delta
\end{aligned}
$$

by Cauchy's inequality.

The estimate (2.13) can be derived by the same arguments using the corresponding result for the process $\underline{X}_{t}^{0, \varepsilon}$.

LEMMA 2.5. For any $\kappa>0$ there exists $\delta_{0}>0$ such that for $0<\delta<\delta_{0}$ there exists $\delta^{\prime}>0$ such that for sufficiently small $\varepsilon$,

$$
\left|P_{\underline{x}}\left(\underline{X}_{\tau_{2}^{\varepsilon}( \pm \delta)}^{\varepsilon} \in C_{2 i}(\delta)\right)-p_{i}\right|<\kappa,
$$

for all $\underline{x} \in D_{2}\left( \pm \delta^{\prime}\right) \cup \partial D_{2}\left( \pm \delta^{\prime}\right)$. Here $p_{1}=\frac{1}{2}$ and $p_{i}=\frac{1}{4}$ if $i=2,3$. 
Proof. Let $T>0$ be small enough that by Lemma 2.3,

$$
\sqrt{A_{7} T}<\kappa / 4 \text {. }
$$

As in the nondegenerate case (see formula (4.26) of [7]), one can get the estimate

$$
E_{\underline{x}} \tau_{2}^{0, \varepsilon}( \pm \delta) \leq A_{10} \delta^{2}|\ln \delta|
$$

for $\delta<\delta_{1}, x \in D_{2}( \pm \delta)$ and small $\varepsilon$ (cf. [6]). The statement of the lemma holds for the process $X_{t}^{0, \varepsilon}$ as shown in [6]. Let $\delta_{0} \leq \delta_{1}$ and $\delta^{\prime}$ be such that for $\underline{x} \in D_{2}\left( \pm \delta^{\prime}\right) \cup \partial D_{2}\left( \pm \delta^{\prime}\right)$,

$$
\left|P_{\underline{x}}\left(\underline{X}_{\tau_{2}^{0, \varepsilon}( \pm \delta)}^{0, \varepsilon} \in C_{2 i}(\delta)\right)-p_{i}\right|<\kappa / 2
$$

and

$$
\frac{2}{T} A_{10} \delta_{0}^{2}\left|\ln \delta_{0}\right|<\kappa / 4
$$

We get for $0<\delta \leq \delta_{0}$,

$$
\begin{aligned}
&\left|P_{\underline{x}}\left(\underline{X}_{\tau_{2}^{\varepsilon}( \pm \delta)}^{\varepsilon} \in C_{2 i}(\delta)\right)-p_{i}\right| \\
& \leq\left|P_{\underline{x}}\left(X_{\tau_{2}^{0, \varepsilon}( \pm \delta)}^{0, \varepsilon} \in C_{2 i}(\delta)\right)-p_{i}\right| \\
& \quad+\left|E_{\underline{x}}\left[\left(I_{0, T}^{\varepsilon}-1\right) \chi_{C_{2 i}(\delta)}\left(X_{\tau_{2}^{0, \varepsilon}( \pm \delta)}^{0, \varepsilon}\right) ; \tau_{2}^{0, \varepsilon}( \pm \delta)<T\right]\right| \\
& \quad+2 P_{\underline{x}}\left(\tau_{2}^{0, \varepsilon}( \pm \delta)>T\right)+\left|E_{\underline{x}}\left[\left(I_{0, T}^{\varepsilon}-1\right) \chi_{\tau_{2}^{0, \varepsilon}( \pm \delta)>T}^{\varepsilon}\right]\right| \\
& \leq \frac{\kappa}{2}+2 A_{10} \frac{1}{T} \delta^{2}|\ln \delta|+\left[E_{\underline{x}}\left(I_{0, T}^{\varepsilon}-1\right)^{2}\right]^{1 / 2} \\
& \leq \frac{3 \kappa}{4}+\sqrt{A_{7} T} \leq \kappa .
\end{aligned}
$$

Here we used the estimates (2.15), (2.16), (2.17), (2.18), Lemma 2.3 and Cauchy's inequality.

ProOF OF THEOREM 1. The proof can be copied from the proof of Theorem 2.2 of [7] (see also the proof of Theorem 1 of [6]). It consists of the following steps. The shape of the operators which govern the limiting diffusion inside the edges is obtained by standard averaging. To get the gluing conditions we use that the Lebesgue measure in $R^{2}$ is invariant for the processes $\tilde{X}_{t}^{\varepsilon}$. For the proof of the Markov property of the limiting process some a priori bounds for the operators $L^{\varepsilon}$ are developed in [6] and, finally, the convergence to the limiting diffusion on the graph follows from the tightness in the weak topology of the processes $\mathrm{Y}\left(\tilde{X}_{t}^{\varepsilon}\right), 0 \leq t \leq T$.

Let us discuss the details. The proof in [7] is based on a series of lemmas denoted there by Lemma 3.1 through Lemma 3.5. Lemma 3.1 of [7] can be used in the same form, and the statement of Lemma 3.2 of [7] can be shown for the 
situation here in the same way. The statement of Lemma 3.3 of [7] follows for the situation here from Lemma 2.2 and from the considerations that followed [see (2.11)]. Lemmas 3.4 and 3.5 of [7] can be replaced by Lemmas 2.4 and 2.5 above.

We mention that by estimate (2.16) the time which the processes $X_{t}^{0, \varepsilon}$ spend in a small neighborhood $D_{2}( \pm \delta)$ of the critical curve $C(1)$ during a time interval $[0, T], T>0$, tends to 0 uniformly in $\varepsilon$ as $\delta$ tends to 0 . By the existence of the uniformly in $\varepsilon$ bounded Girsanov density we get the same property for the processes $X_{t}^{\varepsilon}$. This implies that the time which the limiting diffusion will spend at the vertex during any time interval $[0, T]$ has Lebesgue measure 0 . Thus, neither the values $L_{i} v_{i}(1)$ nor the values $v_{i}(1)$ should appear in the gluing condition.

Next we consider the asymptotic of the mean first exit time from a bounded domain. Let $G$ be a domain in $[-\pi, \pi] \times R$ bounded by trajectories $\gamma_{1}, \ldots, \gamma_{l}$ of the nonperturbed system (1.2), and

$$
\tau^{\varepsilon}=\min \left\{t: \underline{X}_{t}^{\varepsilon} \notin G\right\} .
$$

It follows from Theorem 1 that

$$
\lim _{\varepsilon \downarrow 0} E_{\underline{x} \tau^{\varepsilon}}=E_{Y(\underline{x})} \tau=E_{z, k} \tau,
$$

where $(z, k)=Y(\underline{x})$ and

$$
\tau=\min \{t: y(t) \notin Y(G)\} .
$$

Then the function $v_{k}(z)=E_{z, k} \tau$ is the solution of the boundary problem,

$$
\begin{array}{rlrl}
L_{k} v_{k}(z) & =-1, \quad(z, k) & \in Y(G) \backslash\left\{Y\left(\gamma_{i}\right), i=1, \ldots, l\right\}, \\
v_{k_{i}}\left(z_{i}\right) & =0, \quad\left(z_{i}, k_{i}\right)=Y\left(\gamma_{i}\right), i=1, \ldots, l,
\end{array}
$$

and, if $\mathrm{O}_{2} \in G$, then the gluing condition (2.9) should be satisfied for $v$, and $v_{j}(1)=v_{k}(1), j, k=1,2,3$.

3. The case of linear drift. Let $F(\varphi, k)$, and $E(\varphi, k), \varphi \in[0, \pi / 2]$, $k \in R, \sin \varphi \leq|k|^{-1}$ be the elliptic integrals of the first and of the second kind,

$$
\begin{aligned}
F(\varphi, k) & =\int_{0}^{\varphi} \frac{d u}{\sqrt{1-k^{2} \sin ^{2} u}}, \\
E(\varphi, k) & =\int_{0}^{\varphi} \sqrt{1-k^{2} \sin ^{2} u} d u, \\
\mathrm{~K}(k) & =F\left(\frac{\pi}{2}, k\right), \quad \mathrm{E}(k)=E\left(\frac{\pi}{2}, k\right) .
\end{aligned}
$$


The terms appearing in the diffusion and the drift coefficient of the limiting diffusion in the theorem can be expressed by these functions:

$$
\begin{aligned}
& \int_{-x_{0}}^{x_{0}} \sqrt{2 z+2 \cos x} d x=4 \sqrt{2} \sqrt{z+1} E\left(\frac{x_{0}}{2}, \sqrt{\frac{2}{z+1}}\right), \\
& \int_{-x_{0}}^{x_{0}} \frac{d x}{\sqrt{2 z+2 \cos x}}=\frac{2 \sqrt{2}}{\sqrt{z+1}} F\left(\frac{x_{0}}{2}, \sqrt{\frac{2}{z+1}}\right) .
\end{aligned}
$$

Thus, the diffusion coefficients $A_{i}(z)=S(z) / T(z),-1<z<1$ if $i=1$, and $z>1$ if $i=2,3$, of the limiting diffusion given by (2.8) can be written

$$
\begin{aligned}
& A_{1}(z)=2(z+1) \frac{E\left(x_{0} / 2, \sqrt{2 /(z+1)}\right)}{F\left(x_{0} / 2, \sqrt{2 /(z+1)}\right)}, \\
& A_{i}(z)=2(z+1) \frac{\mathrm{E}(\sqrt{2 /(z+1)})}{\mathrm{K}(\sqrt{2 /(z+1)})}, \quad i=2,3 .
\end{aligned}
$$

In the special case considered in [1], that is, for

$$
b(x, y)=-\kappa y+\gamma,
$$

the limiting diffusion is governed by the operators

$$
\begin{aligned}
\left(L_{1} v_{1}\right)(z)= & \frac{1}{2} A_{1}(z) v_{1}^{\prime \prime}(z) \\
& +\left(\frac{1}{2}-2 \kappa(z+1) \frac{E\left(x_{0} / 2, \sqrt{2 /(z+1)}\right)}{F\left(x_{0} / 2, \sqrt{2 /(z+1)}\right)}\right) v_{1}^{\prime}(z) \\
= & \frac{1}{2} A_{1}(z) v_{1}^{\prime \prime}(z)+\left(\frac{1}{2}-\kappa A_{1}(z)\right) v_{1}^{\prime}(z) \\
\left(L_{i} v_{i}\right)(z)= & \frac{1}{2} A_{i}(z) v_{i}^{\prime \prime}(z)+\left(\frac{1}{2}+(-1)^{i} \frac{\pi \gamma \sqrt{z+1}}{\sqrt{2} \mathrm{~K}(\sqrt{2 /(z+1)})}\right. \\
= & \frac{1}{2} A_{i}(z) v_{i}^{\prime \prime}(z) \\
& +\left(\frac{1}{2}+(-1)^{i} \frac{\mathrm{E}(\sqrt{2 /(z+1)})}{\sqrt{2} \mathrm{~K}(\sqrt{2 /(\sqrt{z+1})})}\right) v_{i}^{\prime}(z)
\end{aligned}
$$


$i=2,3$, and by the gluing condition (2.9) at the point $O_{2}$. The vertex $O_{1}$ is inaccessible.

Let $B_{i}(z)$ denote the drift coefficients [the coefficients of $v_{i}^{\prime}(z)$ in (3.3) and (3.4)]. For the following calculations it is convenient to write the differential operators in the form $L_{i}=D_{m_{i}} D_{s_{i}}^{+}, i=1,2,3$, where $s_{i}$ is the scale function and $d m_{i}$ is the speed measure of the limiting diffusion inside the edges of $\Gamma$ (see [3], [9]),

$$
\begin{aligned}
& m_{i}^{\prime}(z)=\frac{2}{A_{i}(z)} \exp \left\{\int_{1}^{z} \frac{2 B_{i}(y)}{A_{i}(y)} d y\right\}, \\
& s_{i}^{\prime}(z)=\exp \left\{-\int_{1}^{z} \frac{2 B_{i}(y)}{A_{i}(y)} d y\right\}
\end{aligned}
$$

$i=1,2,3$. We calculate the invariant density $\rho_{i}^{\kappa, \gamma}(z),(z, i) \in \Gamma, i=1,2,3$ of the limiting process on $\Gamma$. First we mention that the Lebesgue measure is invariant for the process $\underline{X}_{t}^{0, \varepsilon}$, that is, for $\kappa=\gamma=0$. Thus, the image of the Lebesgue measure under the map $Y$ gives an invariant measure on $\Gamma$ for the limiting diffusion in this case, and $\rho^{0,0}$,

$$
\rho_{i}^{0,0}(z)=c_{i} T(z), \quad(z, i) \in \Gamma \backslash\left\{O_{2}\right\}, i=1,2,3, c_{1}=2 c_{2}=2 c_{3}>0
$$

is an invariant density. The invariant density in the general case can be written

$$
\rho_{i}^{\kappa, \gamma}(z)=c \alpha_{i} m_{i}^{\prime}(z), \quad(z, i) \in \Gamma \backslash\left\{O_{2}\right\}, i=1,2,3, c>0,
$$

where $\alpha_{i}$ is the coefficient in the gluing condition if the gluing condition is written in the form $\sum_{2}^{3} \alpha_{i}\left(D_{s_{i}} u_{i}\right)(1)=\alpha_{1}\left(D_{s_{1}} u_{1}\right)(1)$. Thus $\alpha_{2}=\alpha_{3}=1, \alpha_{1}=2$. Using (3.3), (3.4) and the fact that the result for $\kappa=\gamma=0$ should coincide with (3.7) we get

$$
\begin{aligned}
& \rho_{1}^{\kappa, \gamma}(z)=2 c \frac{2}{A_{1}(z)} \exp \left\{\int_{1}^{z}\left(\frac{1}{A_{1}(y)}-2 \kappa\right) d y\right\} \\
&= 2 c T(z) \exp \{-2 \kappa(z-1)\}, \quad 0<z<1 \\
& \rho_{i}^{\kappa, \gamma}(z)=c \frac{2}{A_{1}(z)} \exp \left\{\int_{1}^{z} \frac{2 B_{i}(y)}{A_{i}(y)} d y\right\}=\rho_{i}^{0,0}(z) \\
& \times \exp \left\{\int_{1}^{z} 2\left((-1)^{i} \frac{\pi \gamma \sqrt{y+1}}{\sqrt{2} \mathrm{~K}(\sqrt{2 /(y+1)})}-\kappa A_{i}(y)\right)\right.
\end{aligned}
$$

$$
\left.\times A_{i}(y)^{-1} d y\right\}
$$




$$
\begin{aligned}
=c T(z) \exp \{- & 2 \kappa(z-1) \\
& \left.+(-1)^{i} \frac{\pi \gamma}{\sqrt{2}} \int_{1}^{z} \frac{d y}{\sqrt{y+1} \mathrm{E}(\sqrt{2 /(y+1)})}\right\},
\end{aligned}
$$

$i=2,3, z>1$. Similar formulas were also given in [1].

Now let $G$ be a domain in $R^{2}$ bounded by the trajectories $\gamma_{1}, \gamma_{2}, \gamma_{3}$ of the nonperturbed system so that

$$
z_{i}=Y\left(\gamma_{i}\right) \in I_{i}, \quad i=1,2,3 .
$$

We consider the problem of mean first exit time from $G$ as described at the end of the foregoing section. We calculate the functions $v_{i}(z)=E_{z, i} \tau$. The functions

$$
v_{i}(z)=c_{i}\left|s_{i}(z)-s_{i}\left(z_{i}\right)\right|+\int_{z}^{z_{i}}\left(s_{i}(z)-s_{i}(y)\right) d m_{i}(y)
$$

$i=1,2,3$, are solutions of the system (2.19), (2.20) for any constants $c_{i}$, $i=1,2,3$. The constants $c_{i}$ can be calculated uniquely from the conditions $v_{i}(1)=v_{j}(1), i, j=1,2,3$, and the gluing condition by solving a linear system for $c_{i}, i=1,2,3$.

We define the functions $K_{i}^{\kappa, \gamma}$,

$$
\begin{aligned}
& K_{1}^{\kappa, \gamma}(z)=-2 \kappa(z-1), \quad 0<z<1 \\
& K_{i}^{\kappa, \gamma}(z)=-2 \kappa(z-1)+(-1)^{i} \frac{\pi \gamma}{\sqrt{2}} \int_{1}^{z} \frac{d y}{\sqrt{y+1} \mathrm{E}(\sqrt{2 / y+1})},
\end{aligned}
$$

$i=2,3, z>1$.

If $\kappa=\gamma=0$ then (3.6) becomes

$$
\begin{aligned}
\frac{d}{d z} s_{i}^{0,0}(z) & =\exp \left\{-\int_{1}^{z} \frac{T(y)}{S(y)} d y\right\} \\
& =\exp \left\{-\int_{1}^{z} \frac{S^{\prime}(y)}{S(y)} d y\right\}=\frac{S(1)}{S(z)}=\frac{8 \mathrm{E}(1)}{S(z)}
\end{aligned}
$$

Thus, in general,

$$
s_{i}^{\prime}(z)=\frac{8 \mathrm{E}(1)}{S(z)} \exp \left(-K_{i}^{\kappa, \gamma}(z)\right) .
$$

Comparing (3.7), (3.8) and (3.9), (3.10) we get

$$
m_{i}^{\prime}(z)=T(z) \exp \left(K_{i}^{\kappa, \gamma}(z)\right), \quad i=1,2,3 .
$$

Let $\alpha_{1}=2, \alpha_{2}=\alpha_{3}=1$,

$$
\Delta s_{i}(z)=\left|s_{i}(z)-s_{i}\left(z_{i}\right)\right|, \quad \tilde{v}_{i}(z)=\int_{z}^{z_{i}}\left(s_{i}(z)-s_{i}(y)\right) d m_{i}(y)
$$


[a solution of (2.19)] and

$$
\rho(G)=\sum_{i=1}^{3} \alpha_{i}\left|\int_{1}^{z_{i}} d m_{i}\right|
$$

[the invariant measure on $Y(G)$ according to (3.8), (3.9), (3.10) with $c=1$ ]. Then (3.11) can be written

$$
v_{i}(z)=c_{i} \Delta s_{i}(z)+\tilde{v}_{i}(z), \quad i=1,2,3,(z, i) \in Y(G) \backslash\left\{O_{2}\right\},
$$

and it turns out that

$$
c_{i}=\frac{1}{\Delta s_{i}(1)}\left(\sum_{j=1}^{3} \frac{\alpha_{j}}{\Delta s_{j}(1)}\right)^{-1}\left(\sum_{j=1}^{3} \frac{\alpha_{j} \tilde{v}_{j}(1)}{\Delta s_{j}(1)}+\rho(G)\right)-\frac{\tilde{v}_{i}(1)}{\Delta s_{i}(1)} .
$$

The relations (3.14) and (3.15) give the general solution of the problem for a diffusion process on a graph if the domain $G$ contains only one vertex (of mass zero for the invariant measure). In the case under consideration we have

$$
\begin{aligned}
& \Delta s_{i}(z)=8 \mathrm{E}(1)\left|\int_{z}^{z_{i}} S(y)^{-1} \exp \left(-K_{i}^{\kappa, \gamma}(y)\right) d y\right|, \\
& \tilde{v}_{i}(z)=8 \mathrm{E}(1) \int_{z}^{z_{i}} \int_{y}^{z} S(x)^{-1} \exp \left(-K_{i}^{\kappa, \gamma}(x)\right) T(y) \exp \left(K_{i}^{\kappa, \gamma}(y)\right) d x d y, \\
& i=1,2,3 .
\end{aligned}
$$

4. Remarks and generalizations. Consider a Hamiltonian system with one degree of freedom

$$
\dot{\mathrm{X}}_{t}=\bar{\nabla} H\left(\underline{X}_{t}\right), \quad \underline{X}_{0}=(x, y) \in R^{2} .
$$

Let the perturbed process $\underline{X}_{t}^{\varepsilon}$ in $R^{2}$, after the time rescaling, be governed by the operator

$$
L^{\varepsilon}=\frac{1}{\varepsilon}\left(\frac{\partial H}{\partial y} \frac{\partial}{\partial x}-\frac{\partial H}{\partial x} \frac{\partial}{\partial y}\right)+b(x, y) \frac{\partial}{\partial y}+\frac{1}{2} \frac{\partial}{\partial y}\left(a(x, y) \frac{\partial}{\partial y}\right) .
$$

We assume, for simplicity, that $H(x, y), b(x, y)$ and $a(x, y)$ are $C^{\infty}$ functions, $a(x, y)>a_{0}>0, H(x, y)$ is generic (see [7]). Let $\Gamma=\left\{I_{1}, \ldots, I_{n} ; O_{1}, \ldots, O_{m}\right\}$ be the graph corresponding to $H(x, y) ; I_{i}$ are the edges and $O_{k}$ are the vertices of the graph (see Figure 2).

Let $Y: R^{2} \rightarrow \Gamma$ be the corresponding map (see [7]). The pairs $(H, i)$ define a global coordinate system on $\Gamma: H=H(u)$ is the value of the Hamiltonian on $Y^{-1}(u), u \in \Gamma ; i=i(u)$ is the index of the edge containing $u \in \Gamma$. Let $C_{i}(z)=Y^{-1}(z, i) \subset R^{2}, G_{i}(z)$ be the domain in $R^{2}$ bounded by $C_{i}(z)$. Put

$$
\begin{aligned}
& T_{i}(z)=\int_{C_{i}(z)} \frac{d l}{|\nabla H(x)|}, \\
& A_{i}(z)=\int_{G_{i}(z)} \frac{\partial}{\partial y}\left(a(x, y) \frac{\partial H(x, y)}{\partial y}\right) d x d y .
\end{aligned}
$$




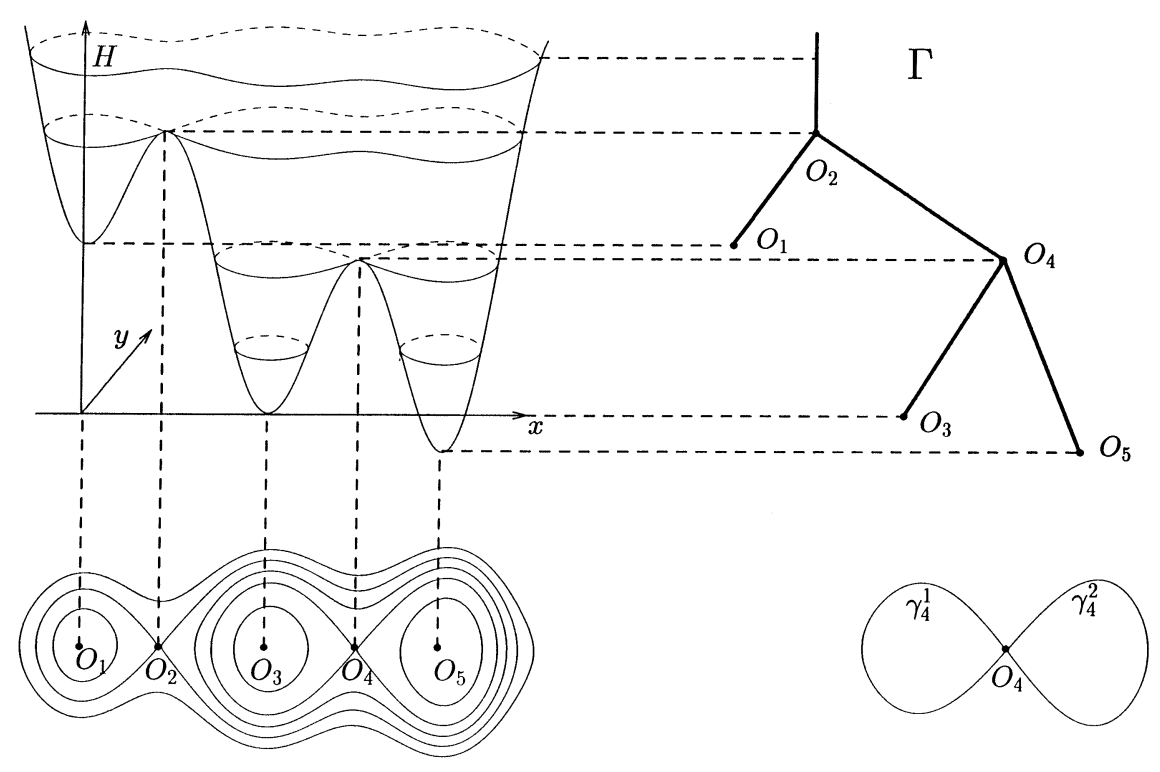

FIG. 2.

Let $O_{k}$ be an interior vertex of $\Gamma$ (corresponding to a saddle point of $H(x, y)$ ). Let $I_{i_{1}}, I_{i_{2}}, I_{i_{3}}$ be the edges attached to $O_{k}$. The set $Y^{-1}\left(O_{k}\right)$ consists of the equilibrium point $O_{k}$ and two trajectories of the nonperturbed system $\gamma_{k}^{1}$ and $\gamma_{k}^{2}$ for which $O_{k}$ is the limiting point as $t \rightarrow \pm \infty$. Let $G_{j}\left(O_{k}\right)$ be the domain bounded by $\gamma_{k}^{j}, j=1,2$. Let $\gamma_{k}^{j}$ be the limiting set for trajectories from $Y^{-1}\left(I_{i_{j}}\right), j=1,2$, and $\gamma_{k}^{1} \cup \gamma_{k}^{2}$ be the limiting set for the trajectories from $Y^{-1}\left(I_{i_{3}}\right)$. Define

$$
\beta_{k j}= \pm \int_{G_{j}\left(O_{k}\right)}\left(a(x, y) H_{y}(x, y)\right)_{y} d x d y, \quad j=1,2 .
$$

The sign "+" should be taken in the definition of $\beta_{k j}$, if the trajectory goes along $\gamma_{k}^{j}$ clockwise, and the sign “-” otherwise. Put $\beta_{k 3}=-\left(\beta_{k 1}+\beta_{k 2}\right)$,

$$
B_{i}(z)=\int_{G_{i}(z)} \frac{\partial b(x, y)}{\partial y} d x d y .
$$

THEOREM 2. The processes $Y\left(\underline{X}_{t / \varepsilon}^{\varepsilon}\right), 0 \leq t \leq T<\infty$, on $\Gamma$ converge weakly in the space of continuous functions as $\varepsilon \rightarrow 0$ to the diffusion process $Y_{t}$ on $\Gamma$, which is defined as follows: inside an edge $I_{i} \subset \Gamma$, the process $Y_{t}$ is governed by the operator

$$
L_{i}=\frac{1}{2 T_{i}(z)} \frac{d}{d z}\left(A_{i}(z) \frac{d}{d z}\right)+\frac{1}{T_{i}(z)} B_{i}(z) \frac{d}{d z} .
$$


A smooth inside the edges $I_{i} \subset \Gamma$ function $f(u), u \in \Gamma$, belongs to the domain $D$ of the generator $A$ of the process $Y_{t}$ if and only if $f(u)$ is continuous on $\Gamma$ together with $A f(u)$, and at any interior (corresponding to a saddle point) vertex $O_{k} \in \Gamma$ the following condition is satisfied:

$$
\sum_{j=1}^{3} \beta_{k j}\left(D_{j} f\right)\left(O_{k}\right)=0,
$$

where $D_{j}$ is differentiation in $z$ along $I_{i_{j}} \sim O_{k}, j=1,2,3$. The operators $L_{i}$ and the gluing conditions (4.3) define the limiting process $Y_{t}$ in a unique way.

The proof of this theorem is a slight generalization of the estimates obtained in [6] and of estimates presented in this paper.

A version of the result holds also for the pendulum equation.

\section{REFERENCES}

[1] Burdzeiko, B. P., Ignatov, Yu. F., Khasminskit, R. S. and Shahgildian, W. W. (1979). Statistical analysis of a model of phase synchronization. In Proceedings of the Fifth International Conference in Information Theory, Tbilisi, USSR, 64-67.

[2] DunYAK, J. and FREIDLIN, M. (1998). Optimal residence time control of Hamiltonian system perturbed by white noise. SIAM J. Control Optim. 36 233-252.

[3] Dynkin, E. B. (1965). Markov Processes. Springer, Berlin.

[4] Feller, W. (1954). Diffusion processes in one dimension. Trans. Amer. Math. Soc. 97 1-31.

[5] Freidlin, M. (1985). Functional Integration and Partial Differential Equation. Princeton Univ. Press.

[6] Freidlin, M. and Weber, M. (1998). Random perturbations of nonlinear oscillators. Ann. Probab. 26 925-967.

[7] Freidlin, M. I. and Wentzell, A. D. (1994). Random perturbations of Hamiltonian systems. Mem. Amer. Math. Soc. 109.

[8] KHAS'MINSKII, R. Z. (1968). Averaging principle for stochastic differential Itô equations. $K y$ bernetica 4 260-279. (In Russian.)

[9] MandL, P. (1968). Analytical Treatment of One-Dimensional Markov Processes. Springer, Berlin.

DEPARTMENT OF MATHEMATICS

UNIVERSITY OF MARYLAND

COLlEGE PARK, MARYLAND 20742
DEPARTMENT OF MATHEMATICS

TECHNICAL UNIVERSITY OF DRESDEN D-01062 DRESDEN

GERMANY 